\title{
Innovative Inertial Measurement Data Acquisition and Processing for Aircraft Surveying up to Airborne Gravimetry
}

\author{
M. Hoss ${ }^{1}$, Dr. rer. nat. A. Dreyer ${ }^{1}$, Dr.-Ing. E. L. von Hinueber ${ }^{1}$, R. Urli ${ }^{2}$, F. Lapeyrade
}

${ }^{1}$ iMAR Navigation $\mathrm{GmbH}$, Im Reihersbruch 3, D-66386 St. Ingbert; +49 68949657620

${ }^{2}$ Airbus Helicopters Deutschland $\mathrm{GmbH}$, Donauwörth

${ }^{3}$ Airbus Operations S.A.S., Toulouse

\begin{abstract}
The paper presents the usage of modern strap-down inertial measurement systems with innovative signal processing within the wide range of applications from highly precise aircraft motion surveying and aircraft testing to airborne gravimetry. A unified hardware and software architecture is shown which allows covering quite different applications like vehicle testing or geodetic surveying with the same platform approach. High dynamics measuring capabilities as well as reliability and accuracy are key requirements for aircraft testing applications, while e.g. quite static flight conditions are the challenge for the missions when determining the Earth's gravity on micro-g level.

Between both applications, which are discussed in the paper, based on practical flight data obtained from a test mission of upgraded $\mathrm{CH}-53 \mathrm{OBS}$ and flight tests for aircraft certification of Airbus A350XWB (and other civil Airbus A/C types like A330neo, etc.), located for most of them over South of Europe, also flight missions from fighter aircraft up to supersonic missile navigation with similar systems of iMAR's iNAT INS/GNSS family are presented as well as the usage of these systems on optical surveying pods for environmental monitoring tasks. Beside these applications, the impact of used inertial sensor technologies like MEMS, fibre-optic or ring-laser gyroscopes is explained.
\end{abstract}

As a counterpart or in addition to airborne surveying with aircraft installed equipment, in the third section of the paper a new generation of ground-based aircraft trackers of type ilPSC-MSG with highspeed and IR cameras for surveying tasks is presented.

Key words: precise dynamics measurement, unified architecture, strap down, inertial, airborne gravimetry, aircraft motion surveying, Airbus civil and military aircraft, iMAR navigation systems, motion tracking, cinetheodolite

\section{Introduction}

Today, a huge amount of different tele-metrical tasks require various levels of inertial precision. These requirements can start at very rough $\mathrm{km}$ precision in WGS84 and go down to sub $\mathrm{mm}$ motion analysis of customer targets, which might be necessary for further correlation of different measuring sensors like vector magnetrometry or gravimetry for example. iMAR therefore developed the iNAT / iXCOM family as an inter-compatible system network consisting of modular devices and control software as well as open protocols for easy adaption in any customer usage. Devices of several sensor classes, from the highest performant free inertial class (so called strategic class) down to industrial or even consumer performance classes, are available and behave transparent for the customer in the same manner. Additionally, lots of different aiding possibilities are given - in most cases GNSS for instance is used to get direct relation to the global world coordinate system. Pressure sensors, magnetic compass sensors and odometer data for example can also be connected and fusioned by the 42+ state extended Kalman filter in real-time. Additionally, lots of different customer data or events can be synchronized and time stamped, also for later post-processing. This allows the user an easy way to switch between several systems of the same family of iNAT / iPRENA, iSULONA, IATTHEMO or iCORUS regarding his requirements.

\section{Differences between inertial measurement systems}

To choose the correct INS, it is important to understand the differences between the different types of inertial sensors. Most common gyroscope sensors at the moment are MEMS based, FOG, RLG and HRG. Accelerometers are mainly MEMS or servo types. Each type of sensor has its benefits and disadvantages, both 
technically and commercially. To better understand those differences, they will be compared in the following in a short overview.

\section{Comparison of different types of gyro sen- sors}

First of all, we should have a quick consideration of gyro's relevant parameters. What kind of parameter do they have, what do they mean and what is the impact on the navigation solution?

Angle Random Walk (ARW) is a stochastic noise effect (generated for example due to manufacturing inaccuracies and other physical impacts) and describes the impact of the noise on the measured angle obtained from the gyro output. It is typically characterized under static condition of the gyro.

Drift or day-to-day bias is the offset to be expected on the data of the gyro after calibration. It is characterized over temperature and other specified environmental impacts.

Bias Stability is obtained as a result of Allan Variance Analysis and characterizes the instability of the gyro bias at static condition (motion as well as temperature). It is typically much lower in value than the day-to-day bias and is used e.g. for data fusion parameterization.

Maximum Rate describes the gyros measurement range of angular rate, a high data output rate mostly corresponds with a high sensor bandwidth. Other important parameters are quantization, linearity, scale factor accuracy and stability, but also group delay and many other parameters.

Table 1 shows an overview of an excerpt of typical gyros parameters.

\begin{tabular}{|l|c|c|c|c|c|c|}
\hline $\begin{array}{c}\text { Type: } \\
\text { iNAT- }\end{array}$ & Gyro Type & $\begin{array}{c}\text { ARW } \\
\text { \%sqrt(h) }\end{array}$ & $\begin{array}{c}\text { Drift } \\
\% \mathbf{h}\end{array}$ & $\begin{array}{c}\text { Bias } \\
\text { Stability } \\
\text { \% h }\end{array}$ & $\begin{array}{c}\text { max. Rate } \\
\% \mathbf{s}\end{array}$ & $\begin{array}{c}\text { SR } \\
\mathbf{H z}\end{array}$ \\
\hline $\begin{array}{l}- \text { RQH } \\
\text { (dif.classes) }\end{array}$ & RLG & $\begin{array}{c}0.001- \\
0.0025\end{array}$ & $\begin{array}{c}0.002- \\
0.0036\end{array}$ & $<0.001$ & 800 & 300 \\
\hline $\begin{array}{l}\text {-RQT } \\
\text { (dif.classes) }\end{array}$ & RLG & $\begin{array}{c}0.0012- \\
0.005\end{array}$ & $\begin{array}{c}0.004- \\
0.01\end{array}$ & $<0.001$ & 400 & 400 \\
\hline -FLSG & FOG & 0.0045 & 0.05 & 0.01 & 500 & 600 \\
\hline -FSSG & FOG & 0.15 & 1 & 0.1 & 500 & 450 \\
\hline -MSLG & MEMS & 0.1 & 5 & 0.1 & 500 & 490 \\
\hline -SLN & MEMS & 0.15 & $3^{*}$ & 0.5 & 400 & 500 \\
\hline -SLD & MEMS & 0.15 & $5^{*}$ & 2.5 & 500 & 500 \\
\hline -SLC & MEMS & 0.26 & $10^{*}$ & 5 & 450 & 500 \\
\hline
\end{tabular}

Table 1: Gyro performance table of different INS (excerpt)

* after algorithm converging under motion with GNSS aiding at const. temperature

Now, if we consider a moving inertial sensor (for simplification just in a simple two dimensional model), we can see that an erroneous angle determination for instance directly would lead to a misinterpretation of position due to dead-reckoning, i.e. if heading would be assumed to be course-over-ground.

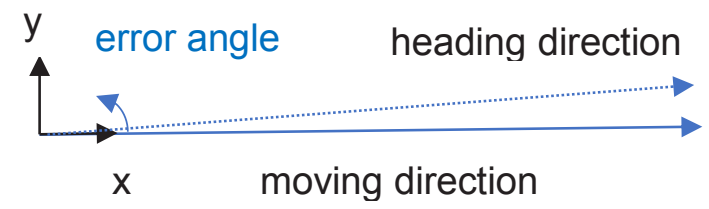

Figure 1: Position error due to dead-reckoning error

This miss-measured angle is caused by the vertical gyro sensor error and even worse, as a gyro does not measure angles but rotation rates, the measured value has to be integrated and the heading error increases over time.

With a constant speed of $50 \mathrm{~m} / \mathrm{s}$ (typical slow aircraft speed) over ten minutes, the position error as a result of dead-reckoning of three different gyro types is compared vs. each other in the next figure. For simplification, only gyro bias instability effects on heading gyro due to dead reckoning are shown.

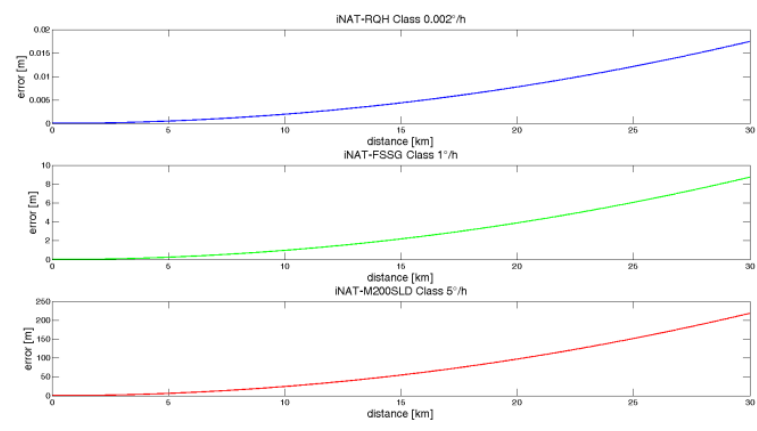

Figure 2: Deflection on a straight line with $50 \mathrm{~m} / \mathrm{s}$ forward motion. Displacement and angle errors over $30 \mathrm{~km}$ (600 s) distance due to gyro drift.

This dedicated effect results in a position error for RLGs of smaller then $2 \mathrm{~cm}$, typical fiber optical gyro of around $10 \mathrm{~m}$ and an industrial range MEMS Gyro of $>200 \mathrm{~m}$ for a distance of $30 \mathrm{~km}$ (600 s).

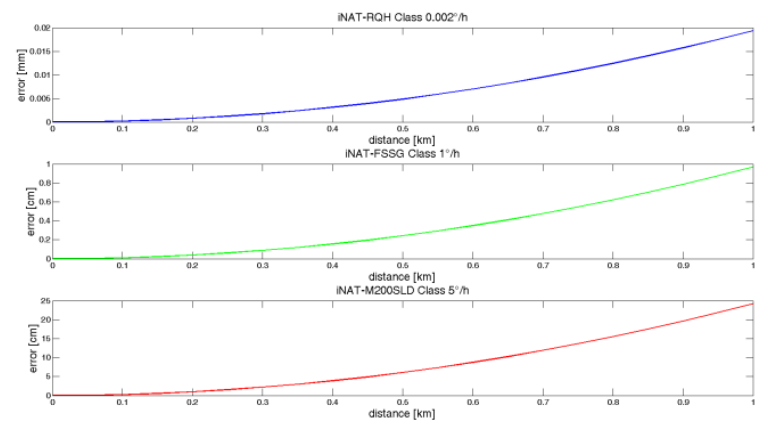

Figure 3: Deflection on a straight line with $50 \mathrm{~m} / \mathrm{s}$ forward motion. Displacement and angle errors over $1 \mathrm{~km}$ (20 s) distance due to vertical gyro drift. 
But, considered for a shorter distance of $1 \mathrm{~km}$ (20 s), this dedicated sensor error generates an error of smaller then $20 \mu \mathrm{m}$ by RLG sensor performance, of around $1 \mathrm{~cm}$ by typical FOGs and of approximately $25 \mathrm{~cm}$ by industrial range MEMS gyros. Of course, real-world deviations are much larger - the here given values shall only show the relation between the different sensor classes.

So, an underrated effect for long term stable free inertial measurements is the gyro bias stability. But what does long term mean? As the effect on position error behaves in an exponential function, the shorter the time period to aid the position measurement the lower the error, but again, the better the gyro stability, the better the solution.

Considering the error for a very small period of time, it is obvious that an angular displacement is very small. In navigation applications, GNSS signals are used as complementary data source to provide position with low data rate, compared to the inertial data, i.e. usually not faster than $10 \mathrm{~Hz}$. Now, even gyroscopes with high drift and instability can be used to interpolate the target position with high data rate between those GNSS based position points with satisfying overall precision, as the increase of the gyro related position error within this period is possibly still far below the error of the GNSS signal itself. It all depends on the requirements of position precision.

Therefore, it is possible to get higher dynamic information $(>10 \mathrm{~Hz})$ due to the higher sample rate of the sensors. This is obligatory to do fast platform controlling for example.

Gyro noise ARW describes the expectable data precision after a specified amount of time. It is an indicator of the statistical error of such a data sample depending on the sample rate of course.

The following figure shows typical values of gyro stability and noise density of different gyro types.

In general, it could be mentioned that for standard navigation, surveying and other precision requiring applications, ring laser gyros have today the most stable bias as well as the lowest noise. MEMS based inertial sensors are still far away from such precision, but became much more precise in the last few years. Fiber optical gyros are in the range between RLGs and MEMS gyros MEMS gyros today even supersede smaller FOGs.

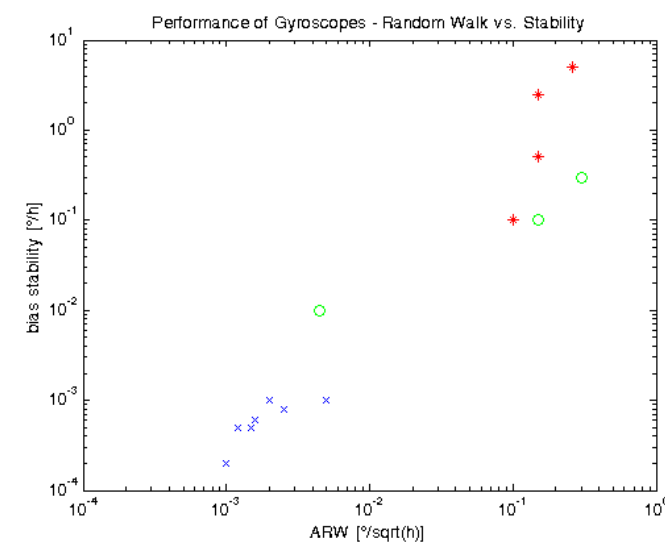

Figure 3: typical Gyro Angle Random Walk vs. stability (examples).

Legend: red +: MEMS, green 0: FOG, blue x: RLG

The high reliability, performance and strong robustness of RLGs even in environment with high vibration and strong temperature gradients compared to the behaviour of FOG and MEMS based sensors has also to be considered in sensor selection. HRG sensors still lack a sufficient availability for the markets described in this paper.

The following table shows the typical impacts.

\begin{tabular}{|c|c|c|c|c|c|}
\hline \multirow{2}{*}{ Gyro type } & \multicolumn{5}{|c|}{ impact with } \\
\cline { 2 - 6 } & Vibration & Temperaure & $\begin{array}{c}\text { Temperat ure } \\
\text { gradient }\end{array}$ & M agnetism & Acceleration \\
\hline RLG & low & low & low & medium & low \\
\hline HRG & low & low & low & low & low \\
\hline FOG & high & medium & medium..high & medium & low \\
\hline MEMS & low...high & high & low...medium & low & medium..high \\
\hline
\end{tabular}

Table 2: Gyro environmental influence dependencies

Another criterion for usage is the quantisation noise for example. RLGs show very high accuracy, but their quantisation is quite high due to the short optical path length. Furthermore, the optical path guiding prism is dithered (mechanical vibration around each sensitive axis with about $500 \ldots 800 \mathrm{~Hz}$, depending on gyro type) to allow a sufficient separation of the two internal optical waves which are required to detect the angular rate based on the Sagnac effect, even at low angular rate. This dithering leads also to significant vibration on the accelerometers mounted close to the ring laser gyros, and therefore the accelerometers have to fulfil certain requirements.

So, each gyro type has its own advantages and hence the design and selection of appropriate gyro technology within an INS/GNSS system needs extended engineering experience.

\section{Accelerometers}

Servo accelerometers are used if navigation grade performance is required (high bias dayto-day performance, low bias drift over time and temperature, accurate scale factor accuracy and linearity, low rectification error etc.), while 
open-loop accelerometers are used for more economical challenging applications with lower performance requirements. Both types are available on MEMS basis, while most accurate navigation grade accelerometers today still are manufactured according the "Q-Flex" principle for many decades now. Manufacturing MEMS based accelerometers with similar performance under similar rugged environmental conditions is a technological challenge, suitable and really comparable devices are expected on the market within the next few years.

But, as a simple engineering example, an accelerometer bias of $1 \mathrm{mg}$ would lead only to a short time position error of $0.01 \mathrm{~m} / \mathrm{s}^{2} \times 0.5 \times 10^{2}$ $\mathrm{s}^{2}=0.5 \mathrm{~m}$ within only $10 \mathrm{sec}$. This explains why a good, navigation grade inertial measurement system usually uses accelerometers with a bias of better than $50 \mu \mathrm{g}$.

\section{Influences on navigation}

As described above, the precision of inertial measurement depends mainly on the precision of gyros and accelerometers. To select the correct types of sensors, it is important to be aware of the requirements one really needs in the specific application. If we need mainly to bridge short GNSS aiding values (position, velocity), a MEMS based INS might be the sufficient choice, because during such short GNSS outages, IMU temperature stays comparable constant and with sufficient motion, the inertial sensor errors can be estimated quite well. On the other hand, if we have to expect long GNSS drop-outs, for example due to strong manoeuvring with significant roll or pitch, due to jamming or any other reason, or if there is the need of very precise RPY angles or position accuracy at any time (e.g. to aid SAR sensors), RLGs are mostly used. FOG based systems are used for performance requirements between both and if no significant temperature gradients and vibration impacts are to be expected (e.g. in subsea environment).

In the following, some typical applications and usages for different types of INS sensors are presented.

\section{Flight data collection of upgraded $\mathrm{CH}-53$}

Airbus Helicopters has been instructed to upgrade the German Forces $\mathrm{CH}-53$ helicopters due to obsolescence of some avionic equipment to a state-of-the-art Helicopter with new flight control and navigation system.

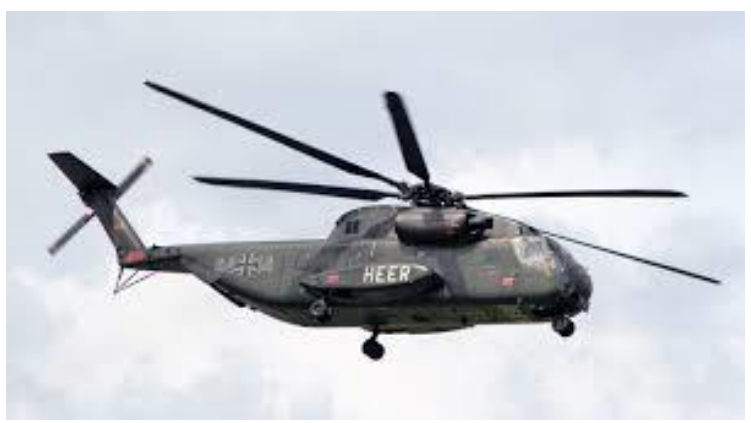

Figure 4: German Forces $\mathrm{CH}-53$ Helicopter.

Airbus Helicopters uses an iMAR iNAT-RQT4003 RLG based INS/GNSS system to evaluate the flight characteristics of al of their helicopters. In the following an example of testing the $\mathrm{CH}-53$ with high accuracy is presented.

These tests took part in March 2020 near Donauwoerth / Germany. Here, an example of a measured trajectory is given.

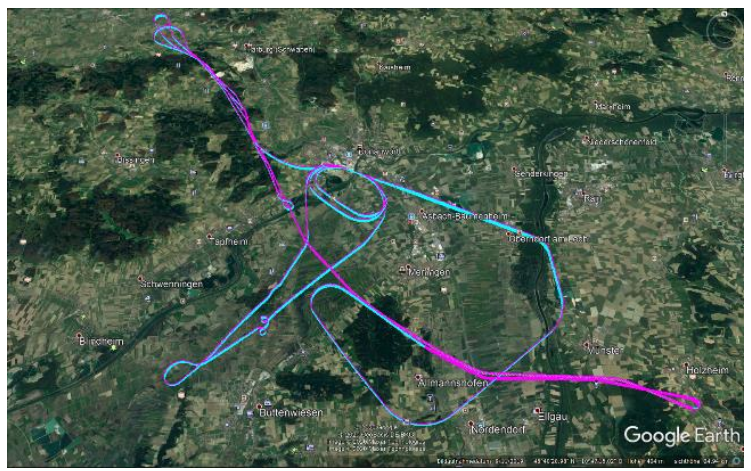

Figure 5: Example trajectories of Airbus Helicopters test flights with $\mathrm{CH} 53$.

\section{Violet: GNSS $1 \mathrm{~Hz}$ Data, Blue: INS 10 Hz Data}

In deeper analysis of this data, different motion of GNSS antenna versus the inertial system can be found. This is explicable as the leverarm between the inertial sensors of the INS/GNSS system and the GNSS antenna is quite huge. Sideward flight and turns are shown impressively in the next figure.

The violet trajectory shows the motion of the GNSS antenna, the blue one the motion of the INS (which is located several meters away from the antenna).

All these data are available in real-time with their maximum rate of $400 \mathrm{~Hz}$ inside the used RLG based iNAT-RQT INS, to feed for example an online flight assistant or to determine limitations of allowed flight parameters for example. Using RTK aiding of GNSS, the 3D trajectory accuracy in real-time is about $2 \mathrm{~cm}$, with an according high accuracy of 3D velocity. 


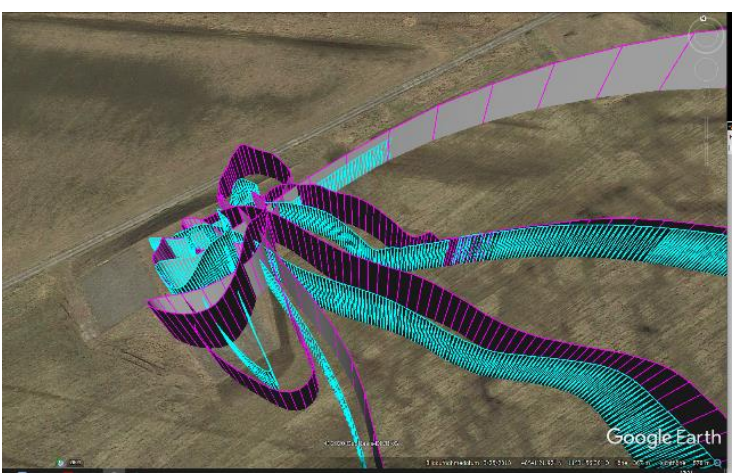

Figure 6: Example trajectories of Airbus Helicopters turn over, rotate and drift manoeuvres (zoom).

Violet: GNSS $1 \mathrm{~Hz}$ Data, Blue: INS $10 \mathrm{~Hz}$ Data

The INS also collects all relevant flight motion data of the helicopter together with precise time-stamps, which can be used for further post processing and to further improve the measurement performance in forward/backward calculation. As an option the iNAT systems can be used as PTP time server to provide accurate time stamping for all other measuring systems on board.

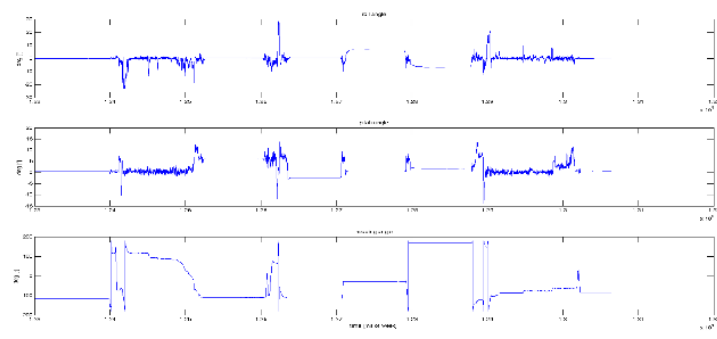

Figure 7: Acquired RPY information of the helicopter.

Additionally, of course, the data can be easily converted and processed with common scientific tools like Matlab etc. to any other format, e.g. to be visualized in GoogleEarth ${ }^{\mathrm{TM}}$. The data can be provided in real-time on several interfaces like Ethernet, UART and CAN, where an individual data decimation (in this case to $10 \mathrm{~Hz}$ from internal $400 \mathrm{~Hz}$ ) and an individual data filtering with user applicable response function can be applied by the flight test engineer. Furthermore, each iNAT INS/GNSS system contains a "black-box" which allows to additionally store all INS/GNSS data (raw and processed data) on non-volatile memory.

Due to the open interface architecture of the iNAT systems, also customer specific data interfaces can be provided, like the Airbus specific IENA protocol, which can be linked into the iNAT standard software and allows delivering systems to customers with dedicated features on request to ease the operation by customer's staff.
The following pictures show a typical integration of the INS/GNSS system - all orange painted equipment and components being mounted on top of it shows flight testing equipment (due to confidentiality reasons the integration into the $\mathrm{CH}-53$ cannot be shown in this paper).

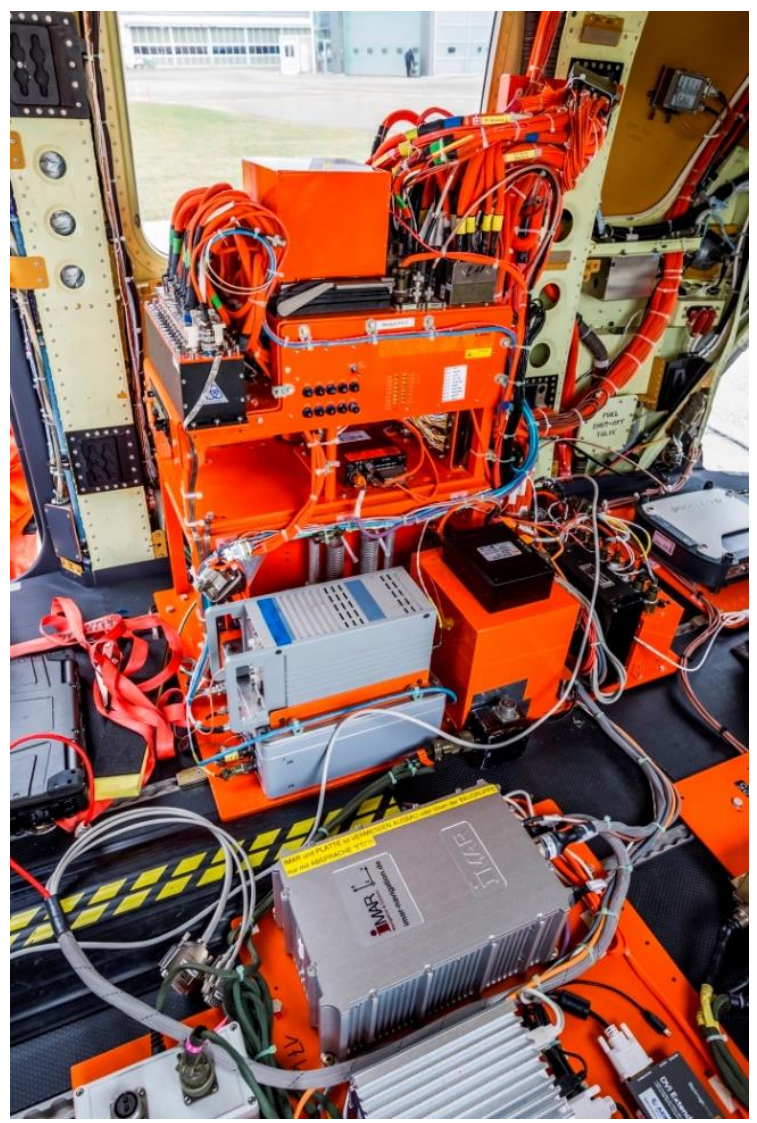

Figure 8: Example of iNAT-RQT integration in $\mathrm{H} 145$ helicopter at Airbus Helicopters.

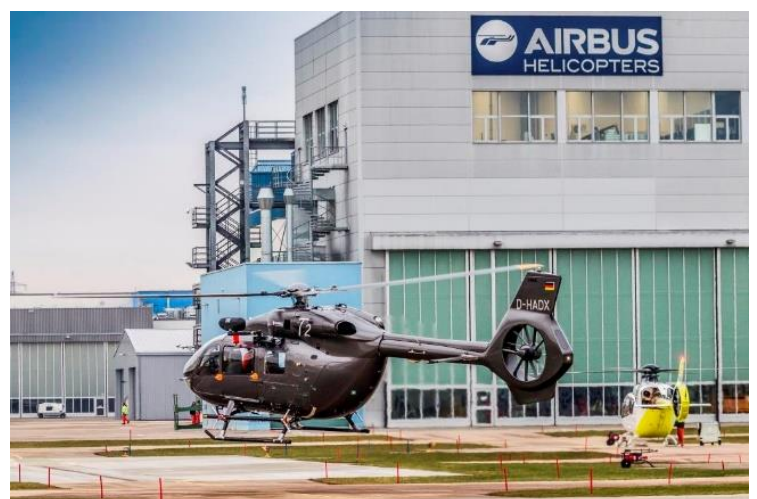

Figure 9: H145 with integrated iNAT-RQT during test flight at Airbus Helicopters.

\section{Monitoring flight tests for aircraft certifica- tion of Airbus A350XWB}

Airbus Operations SAS uses more than half a dozen of iMAR's iNAT-FSLG fibre optic gyro based INS/GNSS systems. They are specifically installed onboard of the development and 
certification aircrafts for performing flight tests for dynamics testing as well as for certification purposes in front of the Airworthiness Authorities (e.g. EASA) of either new air-craft types, new functions or new systems.

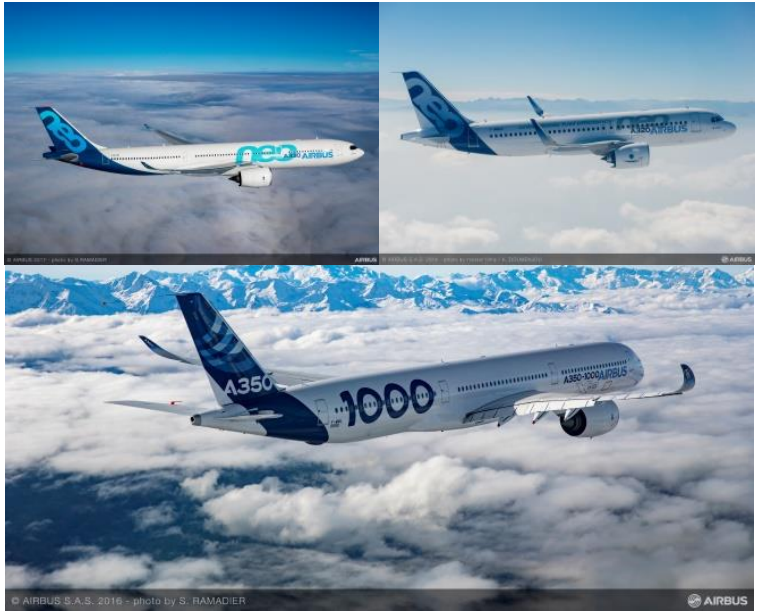

Figure 10: Airbus A320neo, A330neo and A350XWB

The used D0160G qualified iNAT-FSLG-01 contains accurate fiber optical gyros as well as a surveying class all-frequencies / all-constellations GNSS receiver, a sophisticated integrated 42+ state extended Kalman filter, 32+ GByte non-volatile memory as "black-box" for data collection and a full airborne qualified power supply and EMI/EMC filtering. It also contains a new developed L-band based GNSS correction based on TerraStar, supporting world-wide operation.

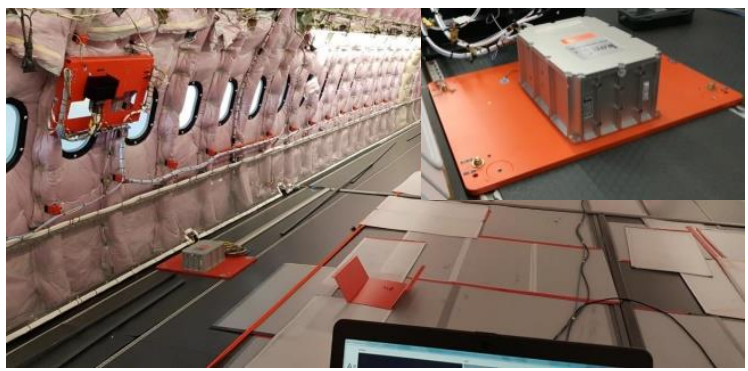

Figure 11: Airbus A330neo single installation

The INS/GNSS system is used mainly for aircraft handling performance testing purposes, both - for real-time guidance used by the flight test engineers and for post-mission data analysis.

iMAR's iNAT-FSLG had been qualified in terms of accuracy towards the $A / C$ certification authorities and performed a demonstration of providing at least the same accuracy or better compared to the previous used traditional INS system.

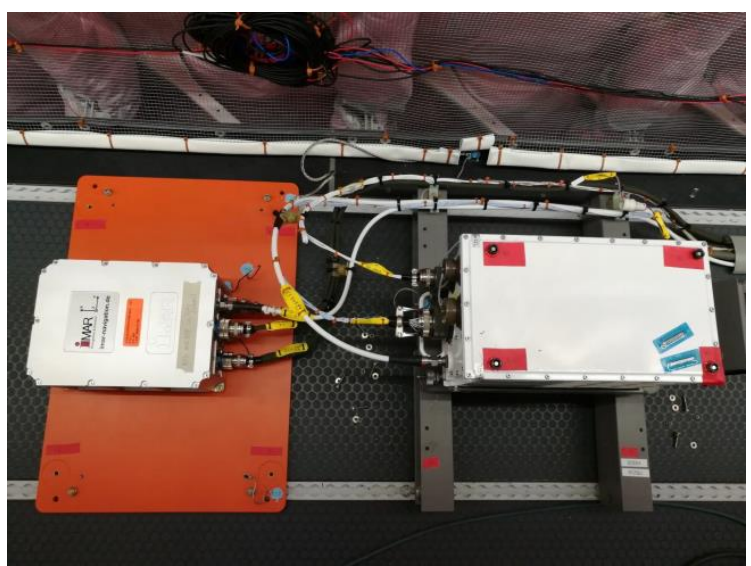

Figure 12: Airbus A330neo back to back installation of light weight iNAT-FSLG (left) and (previous used) standard aviation INS

Since 2018 Airbus Operations SAS uses iMAR's iNAT-FSLG devices in productive operation - on many different types of aircrafts so far.

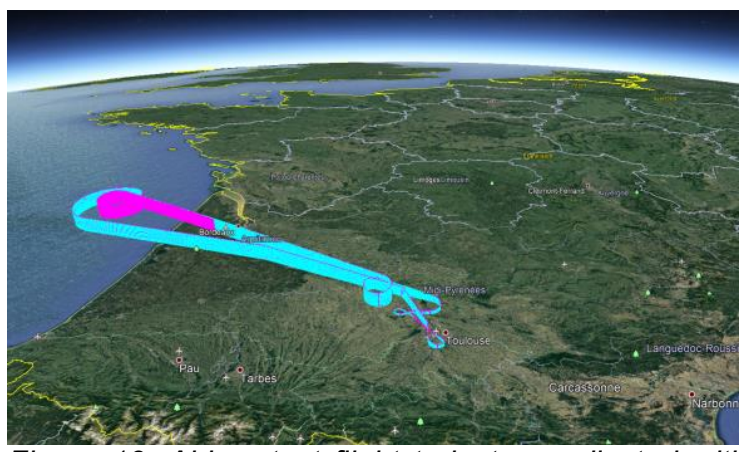

Figure 13: Airbus test flight trajectory collected with iNAT-FSLG in real-time

The following figures show the real-time measured inertial data as well as attitude and velocity. The standard deviations of attitude, heading and velocity show the superior performance of the used sensor system and online hybridization of the INS/GNSS data.

Impressive are the strong pitch angles after take-off during ascending, the significant roll angles during circular flight and the low standard deviations of measured attitude and speed. More detailed data cannot be shown due to confidentiality reasons.

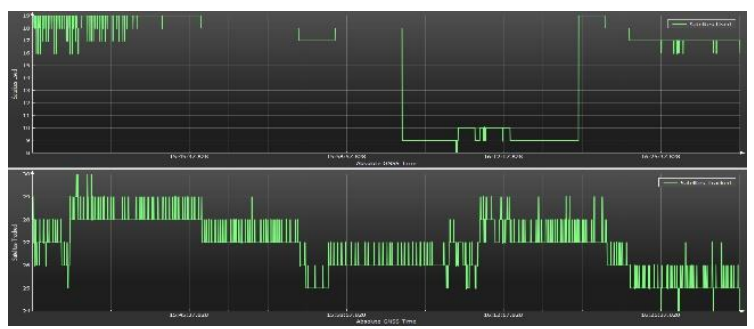

Figure 14: Airbus test flight GNSS satellites used and satellites tracked, correlated to current roll angle 


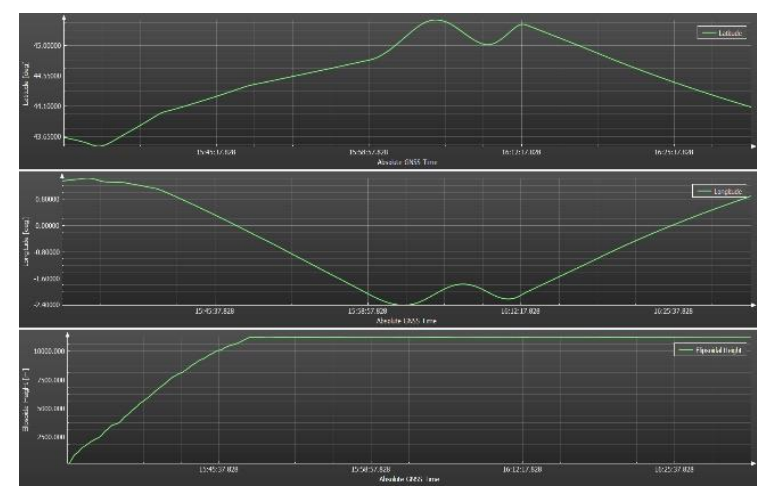

Figure 15: Airbus test flight position and altitude plot (all collected and processed in real-time)

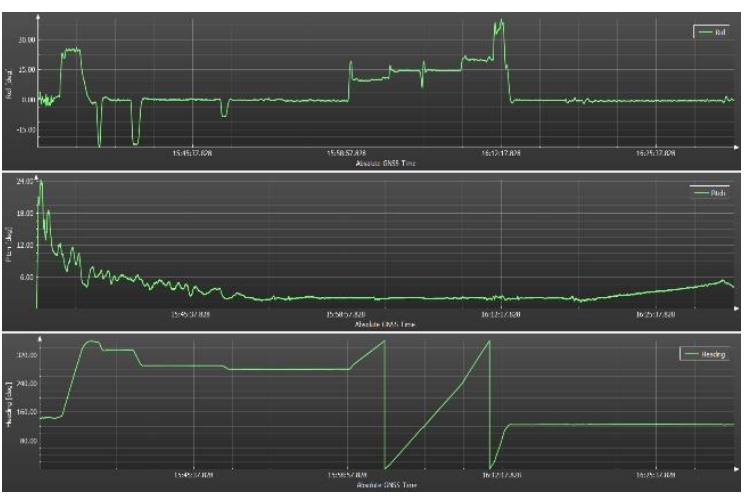

Figure 16: Test flight roll / pitch / heading plot

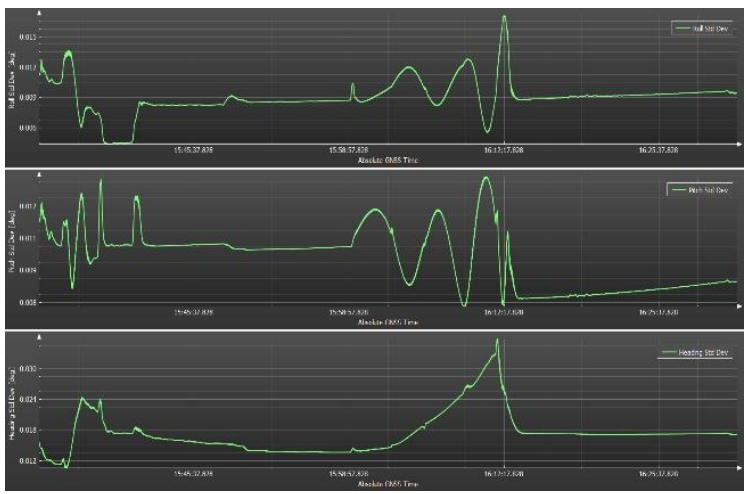

Figure 17: Test flight roll / pitch / heading standard deviations plot

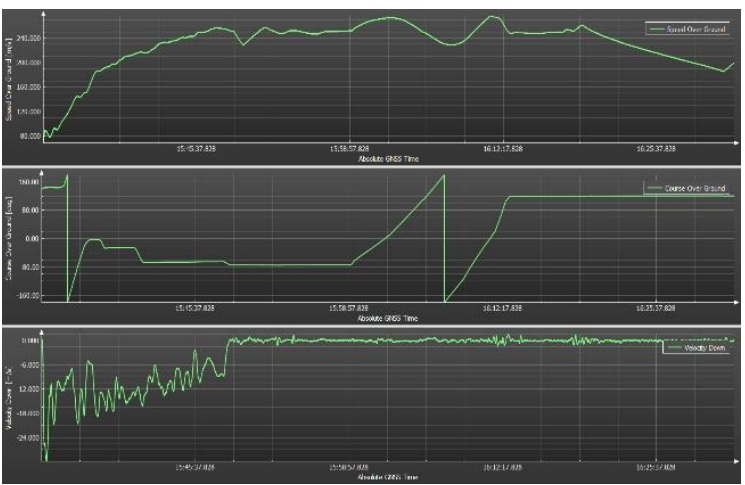

Figure 18: Test flight velocity and course-overground plot

\section{Airborne Strap-Down Gravimetry with high performant RLGs}

Another very interesting application for use of very high performant inertial measurement systems is the discovery of earth's gravity field disturbances. Even most modern gravity field models like e.g. GRACE gravity model still deal with inaccuracies of several meters as their wavelength resolution is above $100 \mathrm{~km}$. To correct these errors, usually 2 axes gimbaled gravimeters are flown in aircrafts to observe the shorter wavelength areas in between.

The idea behind airborne strap down gravimetry is to use a very accurate GNSS signal, which is referenced on WGS84, and a very accurate inertial sensor signal and to subtract their results from each other, including all kind of coordinate transformations, compensations and so on. In general the result should be the difference between both solutions. This difference is the gravity disturbance in WGS84.

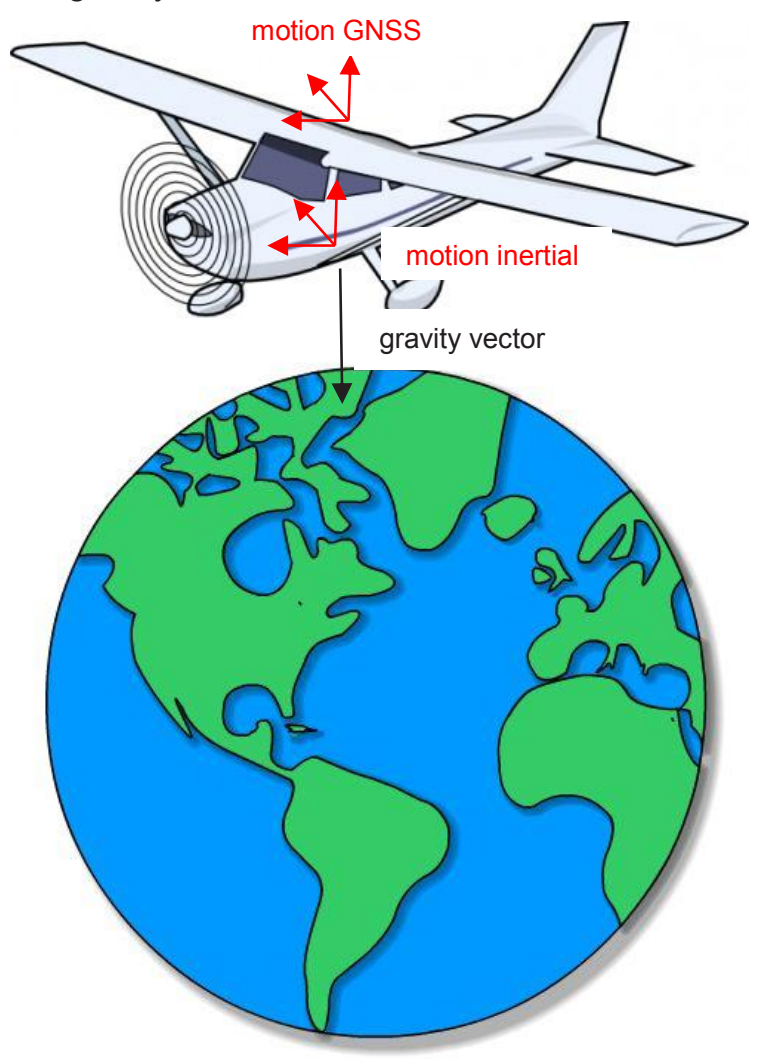

Figure 19: Principle of airborne strap down gravimetry

In simplified words, the GNSS determined motion from the aircraft is removed from the inertial determined motion and only the gravity vector is left. The variation of gravity can be shown in the post processed data. 


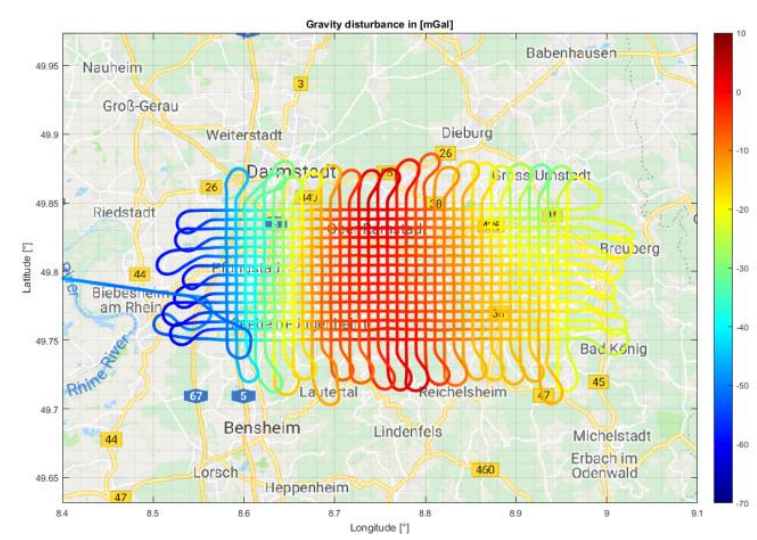

Figure 20: Bouguer anomaly measured south of Darmstadt in iMAR's Odenwald Mission 3/2018

Therefore, the dedicated measurement system iCORUS has been developed considering all significant influences on gyros, accelerometers and timing as mentioned above.

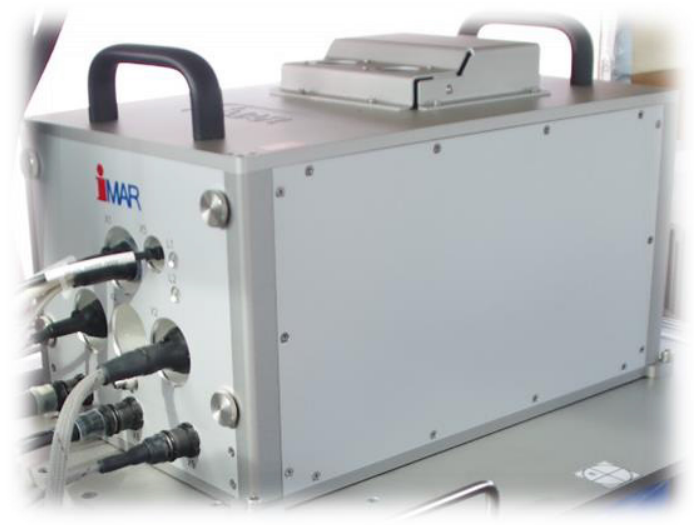

Figure 21: iMAR's iCORUS Gravimeter system

Especially thermal effects are limiting the stability of gyros and accelerometers. Therefore, a highly sophisticated thermal modelling and stabilisation has been developed and applied. Also, most accurate servo accelerometers are used as the distortion of the gravity field is typically in the range of a few mGal only; a value of $1 \mathrm{mGal}$ is the required precision (remember: $1 \mathrm{mGal}$ is equal to $1 \mu \mathrm{g}$ or $10 \mu \mathrm{m} / \mathrm{s}^{2}$ ).

The acceleration stability of this advanced setup within iCORUS has been verified to be better than $0.1 \mu \mathrm{g}$.

As the inertial measurement is to be considered as "free inertial" - at least during the flight lines for surveying - a very accurate gyro design has to be used beside the accelerometers also to minimize position and location errors. For this application, usually gyros with bias significantly better than $0.01 \%$ have to be used together with a dedicated sensor modelling and treatment.

\section{Direct trajectory measurement of flying su- personic objects with iNAT}

While the previous chapters deal with the surveying of the flight behaviour of helicopters and fixed-wing aircrafts, this chapter deals with the question of how to monitor the trajectory and flight behaviour of very fast, small and agile objects. A good example is the request to survey a supersonic missile on a test range. As the obtained results of the flight campaigns in 2018 and 2019 are confidential, the setup shall still be explained. We call this method the "direct trajectory measurement" setup, while the "indirect" method is explained in the following chapter.

For controlling or monitoring supersonic targets, a stand-alone GNSS solution will not be satisfying due to several reasons:

- The GNSS data rate is comparably small: Even fast GNSS receivers provide not more than $100 \mathrm{~Hz}$ data rate

- Taking the GNSS position noise and standard deviation into account as well as the difficulties to obtain continuous GNSS reception on such target, the duration between valid GNSS samples might increase dramatically in real world environment and hence significant information is lost.

- To monitor the behaviour of a supersonic target flight controller or even to feed such controller with relevant data, such data should contain also angular rates and acceleration.

Test flights on a so-called "half scale Pershing" evaluation missile had been performed using iMAR's iNAT-M200/SLN INS/GNSS solution, both for passenger flights as well as for flights where the iNAT-M200 had been used for flight control. The iNAT-M200/SLN is based on MEMS gyroscopes and MEMS accelerometers and an advanced multi-constellation / multifrequency GNSS receiver of latest generation (2019).

The performance of missile, flight controller and iNAT-M200 had been demonstrated, validated and approved at a speed $>2$ Mach. Both the real-time solution of the iNAT as well as all raw data had been transmitted on a radio link to the ground station during the flights. The flight tests confirmed the highly accurate real-time solution of the INS/GNSS system as well as the capability to improve these data in post-processing accordingly. 


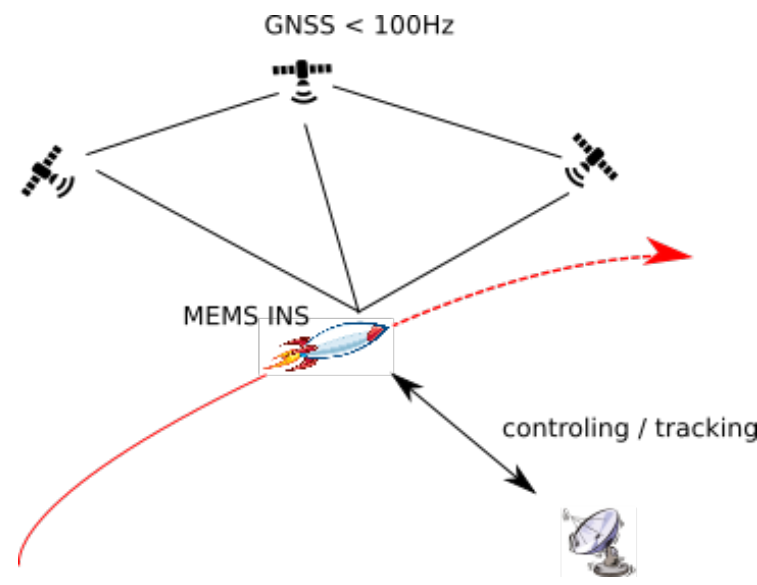

Figure 22: Example of MEMS based INS/GNSS to control or survey trajectories on high speed targets

Besides surveying or control tasks, this setup with the iNAT system is also used to transmit the target's precise location and orientation to a ground station to control the orientation of RF antennas in order to establish a high speed communication link between ground station and target.

\section{Indirect trajectory measurement of flying objects based on optical triangulation}

If there is no possibility to apply an INS/GNSS system on the flying target to obtain the its position and orientation directly as in the previous example, a ground based target tracking has to be considered. This method allows to track flying targets due to indirect triangulation.

With a certain amount of optical tracking platforms with known position and orientation it is possible to determine the target's position in any local or world frame coordinate system.

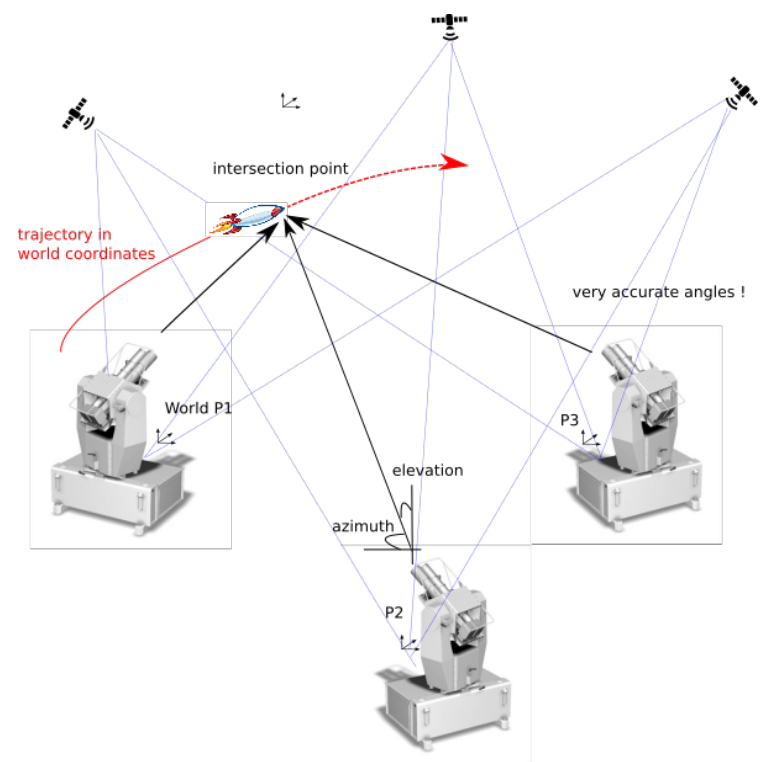

Figure 23: set up of iIPSC-MSG target tracker measuring the trajectory of a moving target
To minimize the statistic errors and to avoid singularities, usually at least a third tracker is used as well. Each optical tracker knows its own position in WGS84 or any other coordinate system from GNSS or geodetic surveying. Due to the integrated INS/GNSS solution and the high resolution encoders, each optical tracker can be operated stationary or applied on a truck or on a naval vessel, and it provides azimuth and elevation of its line-of-sight to the tracked target in real-time. The control centre calculates the three-dimensional trajectory (position and speed) and the estimated future position according to an applicable dynamic motion model of the flying target including its standard deviation.

Several test ranges worldwide are equipped with this equipment. Details are covered by confidentiality of the operators. Published details can be found under the following link:

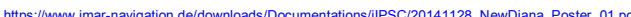

\section{iMAR solution: A system approach}

To provide reliable and accurate inertial measurement systems, a large amount of system and application know-how is necessary.

iMAR's pretention is to give the customer an easy possibility to operate any kind of INS/GNSS solution, optionally extended by magnetometer sensors, air data sensors, odometer etc. with different types and performance classes of sensors within the same toolchain and setup.

To any operator, it does not make any significant difference whether he uses a standard MEMS INS or a high precise RLG INS. All setup, control and data evaluation procedures are similar.

For this purpose iMAR introduced the IXCOM Standard. Any iMAR iNAT device communicates and stores data according to the IXCOM protocol definition. This allows using the same post processing software (iPosCAL), command tools (iXCOM-CMD) and even customer software for any device with the same data interface, independent of the requirements of the application.

iMAR provides off-the-shelf INS as well as customized or OEM versions for an easier integration on customer side:

\section{iNAT / IXCOM - one protocol, one family}

As an open standard, iXCOM can also be implemented in customer applications to allow direct integration. Support tools like Python scripts and an SDK are available. 


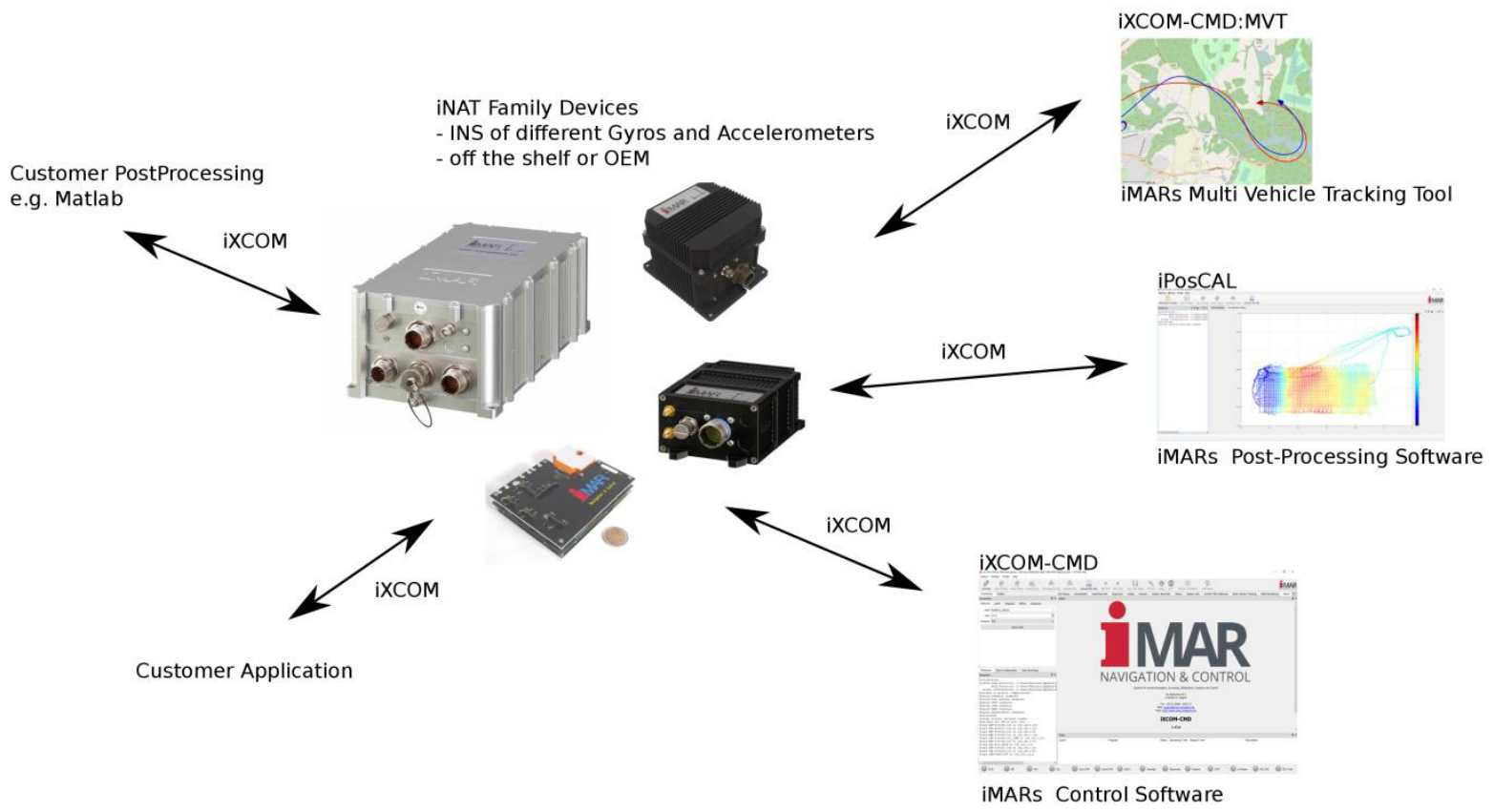

Figure 24: iMAR's iNAT Family Tree with iXCOM interface (excerpt)

Further information can be found under www.imar-navigation.de

\section{Abbreviations}
ARW Angle Random Walk
FOG Fiber Optical Gyro
GNSS Global Navigation Satellite System
INS Inertial Navigation System
MEMS Micro-Electro-Mechanical Systems
RLG Ring Laser Gyro 